\title{
User Interface of Online Chinese-English Dictionary of Traditional Chinese Medicine
}

\author{
Fang $\mathrm{Lu}$ \\ Foreign Language Department \\ Sichuan Vocational and Technical College \\ Suining, China 629000
}

\begin{abstract}
Web crawling, Wiki and other technologies are the necessary measures for compilation of online dictionaries, but rigorous and scientific meaning is still the core of dictionary. In this paper, I selected two examples, "blood heat" and "qi stagnation", from two online Chinese-English dictionaries of Traditional Chinese medicine (TCM), trying to compare and identify the strategy for compiling the entries of online Chinese-English dictionary of TCM.
\end{abstract}

Keywords-non-Chinese learners; CHINESE-ENGLISH dictionary of TCM; analysis of user interface

\section{INTRODUCTION}

With the spread and gradual popularity of TCM culture in the world, more and more people look forward to learning the terms and concepts of TCM, and immediately understand the relevant dialectical and treatment methods via smart phones, I pad and other mobile Internet terminals that can be used offline. To meet such demands of this era, the online Chinese-English dictionaries of TCM came into being. They are created by duplicating printed Chinese-English dictionaries, or through capturing web pages. However, both the former and the latter can not seem to satisfy the users' demands due to lack of preparation. In this paper, I selected two examples, "blood heat" and "qi stagnation " , from two online Chinese-English dictionaries of Traditional Chinese medicine (TCM), trying to compare and identify the strategy for compiling the user interface of online Chinese-English dictionary of TCM.

\section{ANALYSIS OF USER INTERFACES OF TWO ONLINE ChINESE-ENGLISH DiCTIONARIES OF TCM - TAKING "BLOOD HEAT" AND "Qi STAGNATION" AS EXAMPLES}

As the Wikipedia, Wikitionary and other online dictionaries could not provide a sufficient coverage of the TCM terms, such as "blood heat" and "qi stagnation", I selected two on Chinese-English dictionaries of TCM for comparison, namely Modern Chinese Medicine Dictionary and online Chinese Medicine Paradigm Dictionary, to compare the content of two entries. Online Chinese Medicine Paradigm Dictionary is converted from Wiseman's Practical Chinese Medicine Dictionary (1998) , containing 30,000 terms, and Modern Chinese Medicine Dictionary is compiled by Zhu Xingguo. The user interface is as below:

A. blood heat Xue re (blood heat) is a traditional Chinese medicine term. Xue re literally means "blood heat". It is also called xue zao.

The syndrome:

The patient may exhibit thirst, dark urine, dry or hard stools, red tongue or tongue with red tip, yellow coating on tongue, flushed face, acne, thin pulse and/or a rapid pulse. The patient may have a higher than normal body temperature if he has a rapid pulse rate; he/she may also dislike warm temperatures.

B. qi stagnation (氣滯)

Syndromes:

heaviness and distention in chest,

distention feeling or lump in the abdomen, abdomen, dislikes pressing,

irritable

dry bound stool,

rough skin and nails,

dry mouth,

dark red tongue possibly with purple speckles,

purple lips,

amenorrhea or pain during menses,

color of blood of menses is dark with pieces,

purple tongue or with purple marks,

deep stringy pulse.

Usually qi stagnation (qizhi) also results in blood stasis .

Also see liver qi stagnation.

$(08 / 01 / 2016)$ 
A.

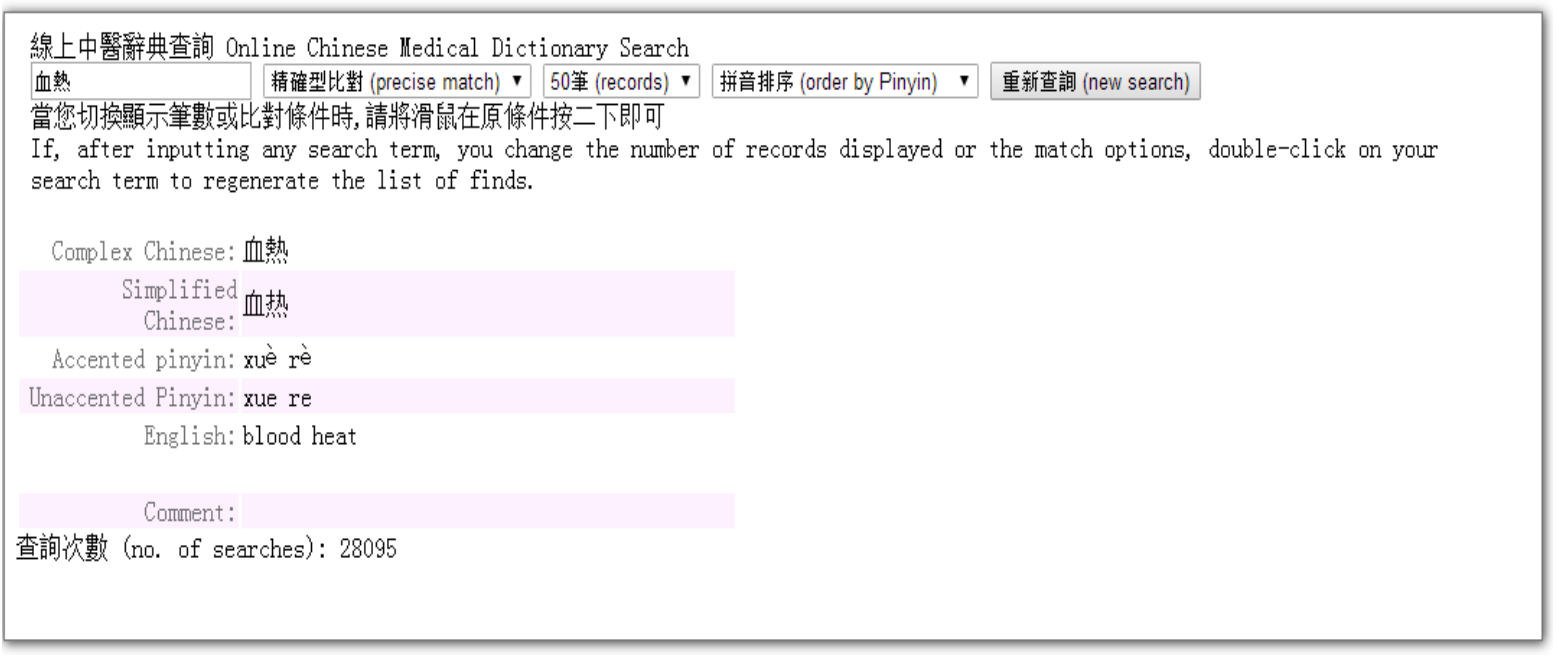

B.

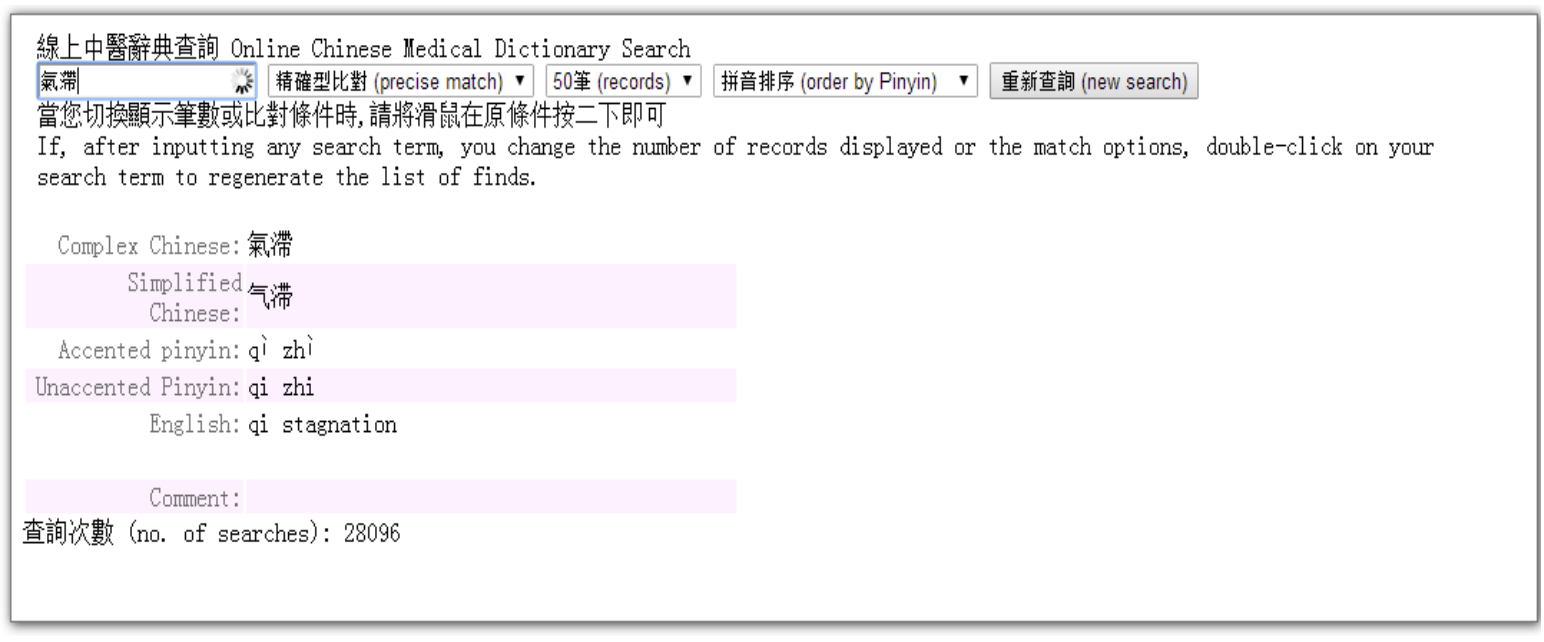

—Online Chinese Medicine Paradigm Dictionary (11/06/2017)

In the user interface of Online Chinese Medicine Paradigm Dictionary, the compiler only provides the corresponding English equivalents of the headwords. In contrast, in addition to the English equivalents, plenty of materials are added in the Modern Chinese Medicine Dictionary. In the entry of "blood heat", some long sentences are used to describes the symptoms of "blood heat". However, the statement used in the interpretation "Xue re (blood heat) is a traditional Chinese medicine term" is obviously redundant, and "he / she may also dislike" and other vocabulary is also tedious, cumbersome and colloquial, which does not meet the norms of dictionary compiling methods. In "qi stagnation", a variety of phrase patterns are used to describes the symptoms. For example, Lines 1, 4, 5, $6,7,8,9$, and 12 are noun phrases; Line 3 is adjective one; Line 10 is a simple sentence; Line 11 contains a noun phrase and prepositional one.

It can be seen that, the user interface of online Chinese Medicine Paradigm Dictionary has not showed a distinguishing feature from the printed ones, in which only the Chinese pinyin and English equivalents are provided, similar to the approach of a glossary. The users have not grasped the cultural and technical concepts, but more confused. As for the Modern Chinese Medicine Dictionary, even though more texts are used to interpret the headwords, the compilers have not kept the same norm to explain the concepts or describe the symptoms. It seems that the entries are created by web crawling. From the discussion mentioned above, we can see that the compilers of two dictionaries do not only have a unified understanding of the dictionaries' user interface, but also lack of scientific compiling approaches. In the two dictionaries, it seems that the users have scarcely enjoyed the benefits brought by the online dictionaries. 


\section{USER INTERFACE DESIGN IN CHINESE-ENGLISH DICTIONARY OF TCM}

For TCM is rooted in the traditional culture of China, it is thought inevitably difficult to write a Chinese-English TCM dictionary. However, it is a necessity to promote the TCM culture to spread around the world. In order to accelerate the transmission of TCM culture throughout the world, it is in an urgent need to write a non-Chinese learners' online ChineseEnglish dictionary of traditional Chinese medicine so as to meet the needs of English users. The dictionary had better be written by Chinese experts or through cooperation of Chinese and English TCM researchers, which aims at English users, available online and offline. For the users, the TCM culture contain a great number of concepts and theories with diverse languages and cultural differences, which are profound and ambiguous. Besides, there are numerous technical terms such as Yin, Yang, Wu Xing, Zangxiang, Qi, blood, fluid adn channels. The concepts are independent and depend upon one another naturally as well, which are deeply rooted in traditional Chinese culture, forming a basic framework for the traditional Chinese medicine theory. Huang Jianhua (2003) holds the view that the bilingual dictionary should be focused on translation but not explanation. However, the translation should be focused on the concept, instead of the individual words. In order to facilitate the deep understanding of the users, the dictionary should contain not only English equivalents as usual, but also some cultural concepts, examples and some multimedia as well. Surely, a highlight for the online dictionary, hyperlink, is also a necessity.

\section{A. Cultural information}

Traditional Chinese medicine has been deeply influenced by Chinese culture. Therefore, cultural information is an indispensable part in some entries. However, the dictionary entries should contain not only some Chinese culture information, but also TCM knowledge. fdx

Consequently, when editing the cultural texts, we should keep the words scientific as well as informative, and the explanations should be specific but not too tedious. Compared with printed dictionaries, online ones could have more storage space. However, the larger storage space could not be identical to the present space. Too many explanations will make the users feel exhaustive, occupy more storage and cause too long for buffer of the App. Therefore, the entries for online dictionaries still should be brief and concise as well, just containing basic concepts. Perhaps the contents vary with font design, but perhaps one full screen could achieve a better effect.

For example, Wu Xing (five elements) has cultural explanations as follows:

The Five Elements theory asserts that between each of the elements there exist the close relationships of mutual generation and mutual controlling. The order of mutual generation among the five elements is that wood generates fire, fire generates earth, earth generates metal, metal generates water, and water generates wood. In this way generation is circular and endless. According to the order of mutual controlling, however, wood controls earth, earth control water, water controls fire, fire controls metal and metal controls wood.

\section{B. Examples}

Exampling is an effective method to show how the entries are grouped with other words to form linguistic units, which is an extension of definition, so it can be integrated to the user interface. For the users, the online Chinese-English dictionary of TCM is cultural and scientific, which is similar to a learner's dictionary to some degree. Therefore, users hope to get more references to help them understand and select therapies of TCM. Less examples could be presented on the user interface, with more authentic data stored in the back end system. If the users would like to refer to more examples, they only need to press the button E.

\section{Multimedia Audio \& Video}

Multimedia audio and video in online dictionaries are just like illustrations in printed dictionaries, which are optional but efficient as references for explanations. They can be illustrations, audio or video documents. Just clicking the multimedia button, $\mathbf{M}$, in the interface, users can have the access to the references of Chinese entries. Besides, some headwords which have the same or similar equivalents, but different designations, such as Futu and Jiexi of acupuncture points, could be displayed in this part. In the meantime, it may help users visually understand some treatment methods exclusively in TCM, such as Aitiaojiu (moxa stick moxibustion) and Baguan (cupping)

\section{Hyperlink}

Hyperlink in online dictionaries is similar to "crossreference" in printed dictionaries. Yet it is not a simple oneone mapping but one-more mapping and an extended "crossreference". Online dictionaries owe the same mediostructure as that of printed ones, in which " cross-reference" functions as the same as that of printed ones. Furthermore, it is a "cross-reference" to the corpus -----the resource of raw materials for the online dictionaries, as well as to the whole Internet. Due to the limitation of the space, only Chinese Pinyin, English equivalent, English definitions and a few examples are presented on the screen. More examples have to be added into hyperlinks. If users need more examples, just click the button E. The design can not only simplify the user interface but also save time, providing more options for the users. Some TCM websites, represented by the button $\mathbf{R}$, can also be added into hyperlinks to supplement the entries. Though Wiktionary and Wikipedia have been widely used, they can not provide an exhaustive coverage of TCM entries. Therefore, they can be adopted as a hyperlink to meet the needs of some Chinese beginners. Dictionaries, such as Iciba, Youdao and Bing, have a comparatively mature design, yet they contain a great number of Chinese words, catering to the native Chinese. General non-Chinese learners feel it hard to read, so they can be used an essential part of hyperlinks to meet the immediate and advanced Chinese learners' demands. 
Though online dictionaries can have the same capacity of entries as printed ones, what it gives users is only an interface. And the users are unable to grasp the macrostructure in a short time. Therefore, in order to attract the users' eyeballs, more attentions shall be paid to the users interface. By comparing and analyzing the user interface in TCM dictionaries at home and abroad, I hereby try to present the user interface of the two samples, blood heat and Qi stagnation as follows:

\section{血热[xuè rè $]$ blood heat}

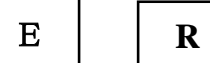

a type of fever which generates heat and flames into the blood aspect, and often causes bleeding, usually recurs daily at noon and subsides in the evening

Eg :

Vaginal bleeding is caused by blood heat.

Blood heat may disturb the mind.

\section{气滞[qì zhì] qi stagnation}

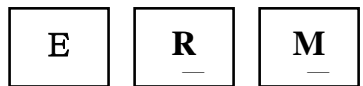

a pathological change characterized by obstructed circulation of qi, manifested as distension or ache in the affected part

Eg:

Static blood may be caused by either long-term qi stagnation or by traumatic injury.

Lower back pain due to qi stagnation makes one difficult to bend forward and backward.

Note: $\mathrm{E}=$ cases $\quad \mathrm{R}=$ Online document hyperlink $\mathrm{M}=$ Multimedia data

Here we can also follow the convention and put the buttons a the top of the interface.

\section{CONCLUSION}

In the internet, though users can get more contents beyond the dictionaries, yet due to the opening writing principle of Wiki series, the accuracy of the dictionaries cannot be guaranteed. Hence, the dictionaries which are written and designed by lexicographers still play an important role. Strict and scientific entry editing is still the core of the dictionary compilation. When designing the user interface of online TCM Chinese-English dictionaries, compiler should consider users' purpose, as well as their thinking and reading habits. Only by this way, can we successfully compile an effective and applicable online TCM dictionaries.

\section{ACKNOWLEDGMENT}

I am appreciative of Chen Congmei, Professor of Southwest University of Science and Technology, for her instructions and comments during the writing of this article and of the financial aids by Sichuan Foreign Language Literature Research Center Project "Research and Writing of TCM Chinese-English Dictionaries with Language Database
Support" (Project No: SCWY15-24) and Special Project for Talent Training in West Regions of China Scholarship Council (LJF[2014] 5049).

\section{REFERENCES}

[1] Philip Durkin ed, The oxford handbook of lexicography. Oxford Oxford University Press, 2015.

[2] Proceedings of eLex 2015. https://elex.link/elex2015/conferenceproceedings/ 08/09/2015.

[3] Sidney I. Landau, Dictionaries: The Art and Craft of Lexicography. Cambridge : Cambridge University Press, 2001.

[4] Sylviane Granger \& Magali Paquot. Electronic Dictionary. Oxford: Oxford University Press, 2012

[5] 08/01/2016.http://www.paradigm-pubs.com/termlist.

[6] 08/01/2016. http://alternativehealing.org/blood_heat_symptoms.htm.

[7] 08/01/2016. http://alternativehealing.org/qi_stagnation.htm.

[8] Cai Yu, Studies on Translation of Traditional Chinese Medicine on the Basis of Recreational Theory [D]. Anhui University, 2013.

[9] Fang Lu, Chen Congmei, Comparison between Yin and Yang Used in Four Chinese-English Dictionaries of Traditional Chinese Medicine [J]. Journal of Southwest University of Science and Technology (Philosophy and Social Science Edition), 2014(6):81-84.

[10] Fang Lu, Entries Setting for Outward Chinese-English Dictionaries of Traditional Chinese Medicine---on the basis of triple heater [J]. Journal of Sichuan Vocational and Technical College, 2014(6): 64-66

[11] Huang Jianhua, Chen Chuxiang, Introduction to Bilingual Dictionaries [M]. Shanghai: The Commercial Press, 2003.

[12] WHO (Western Pacific Region), WHO International Standard Terminologies on Traditional Medicine in the Western Pacific Region[M]. Beijing: Peking University Medical Press, 2009.

[13] Zhang Xiangming, New Development Trend of Electronic Lexicography Studies in the 21st Century-plus comments to Electronic Lexicography [J]. Dictionaries Studies, 2014(2):10-19.

[14] Zhang Yihua, Computational Lexicography and New Types of $\begin{array}{lll}\text { Dictionaries } & {[\mathrm{M}] \text {. }} & \text { Shanghai: }\end{array}$ Lexicographical Publishing House, 2004.

[15] Zhang Yihua, Rethinking of Contents and Nature of Explanations in Bilingual Dictionaries $[\mathrm{J}]$. Journal of Guangdong University of Foreign Studies, 2005(B11):7-10.

[16] Zhou Yibin, Studies on Perspectives and Methods of Traditional Chinese Medicine English Translation Theories on the basis of analysis of core periodicals (D). Nanjing University of Chinese Medicine, 2013.

[17] Nigel Wiseman, Dictionary of Chinese Medicine, Changsha: Hunan Science \& Technology Press, 2007.

[18] Fang Tingyu, et al, New Chinese-English Dictionary of Traditional Chinese Medicine, Beijing: China Medical Science Press, 2013

[19] Li Yongan, English-Chinese Western Medicine-Chinese-English Traditional Chinese Medicine Dictionary, Beijing: Beijing Institute of Technology Press, 2010.

[20] Li Zhaoguo, A Concise Chinese-English Dictionary of Yellow Emperor's Canon of Medicine, Beijing: People's Medical Publishing House, 2011.

[21] Shuai Xuezhong, et al, Chinese-English terminology of traditional Chinese medicine, Changsha: Hunan Science \& Technology Press, 2008.

[22] Xie Zhufan, Classified Dictionary of Traditional Chinese Medicine (New Edition), Beijing: Foreign Language Press, 2002. 